\title{
Incorporating Inter-System Bias in Single Point Positioning Based on GPS, Galileo and BeiDou System
}

\begin{abstract}
The increasing number of satellites provides new opportunities. In the experiment presented in this paper, the Single Point Positioning technique is tested. Data from four different receivers were used in the tests. The GPS, Galileo and BeiDou System observations were collected over a three day long observational session. The computational process was carried out using self-made software and point positions were obtained as the result. The goal of the test was to verify the impact of the Inter-System Bias (ISB) on the final results. For this purpose, two cases of processing data were compared: with estimating ISB and without taking into account this parameter. In the paper the formulas of the mathematical models used are presented and, in both of the considered cases, a combination of GPS, BDS and Galileo was used. The results show that in the case where the ISB was taken into account, the accuracy and precision in the positioning was much better than in the approach where the ISB was not considered. Estimating the ISB allows for more precise positioning results to be obtained for car-navigation or GIS purposes.
\end{abstract}

Keywords: GPS, BDS, Galileo, SPP, ISB

Received: 2 September 2020; accepted: 6 November 2020

(C) 2021 Authors. This is an open access publication, which can be used, distributed and reproduced in any medium according to the Creative Commons CC-BY 4.0 License.

University of Warmia and Mazury, Faculty of Geoengineering, Olsztyn, Poland, email: dawid.kwasniak@uwm.edu.pl, ORCID ID: https://orcid.org/0000-0001-6144-237X

2 University of Warmia and Mazury, Faculty of Geoengineering, Olsztyn, Poland, email: slawomir.cellmer@gmail.com, ORCID ID: https:/orcid.org/0000-0002-2614-8017 


\section{Introduction}

For many years, there were only two operating Global Navigational Satellite Systems (GNSS) - GPS and GLONASS. However, in recent years, two new satellite systems have appeared: the Bei Dou System (BDS) and the Galileo. Galileo is predicted to be fully operational by 2020 . However, it is not certain whether constellation will be ready in 2020. BDS will became fully operational in 2020. All satellites of this system are already in space, however some of them are still in their test phase. In 2012, BDS became operational above the eastern part of Asia [1-3]. Both BDS and Galileo are currently widely used in positioning [4-13]. The Single Point Positioning (SPP) is a well know method. It has been used for navigation since the 1980s. For many years, the accuracy of SPP method was limited to tens of meters, however, this has increased in recent years. This is mainly thanks to improvements such as more precise civil code C/A and more accurate ephemeris [10]. The level of $2-3 \mathrm{~m}$ is a current accuracy of SPP [14]. This does not change the fact that the SPP method it is still in use $[11,14,15]$. The increasing number of GNSS satellites has the potential to improve the accuracy of the SPP method.

\section{GPS, BDS and Galileo Systems Description}

The GPS System is an American navigational system created during "Cold War". The system became fully operational in 1993 with 24 satellites. Nowadays, GPS operates with so called "Expandable 24", which means that the full constellation consists of 27 satellites [2, 16]. However, most of the time over 30 GPS satellites are available for users. The GPS satellites are placed on six orbital planes at an altitude of approximately $20,200 \mathrm{~km}[17,18]$. The inclination for the GPS satellites planes is $55^{\circ}$ [17]. The GPS system uses the following frequencies: L1, L2 and L5 [11]. The frequencies in megahertz [MHz] are shown in Table 1 at the end of Chapter 2. The GPS consists of three segments: the space segment which has already been descripted above; the control segment which consists of a global network of ground facilities that track the GPS satellites, monitors their transmissions, performs analyses, and sends commands and data to the constellation a third segment which is the user segment. Currently, the number of GPS satellites oscillates between 30 and 32 .

The BeiDou System (BDS) is a Chinese navigational system and has been operational above the East Asia region since December 27 2012. The BDS full constellation will consist of 35 satellites: 5 geostationary satellites (GEO), 3 Inclined Geosynchronous Orbits (IGSO) and 27 medium-Earth orbits (MEO) [19, 20]. All of these satellites are currently in space. For the IGSO and GEO satellites the altitude is approximately $35,786 \mathrm{~km}$ when for the MEO satellites the approximated altitude is $21,528 \mathrm{~km}$. For both MEO and IGSO the inclination is $55^{\circ}$ [3]. For the MEO orbit planes period is 
a 12 h 53 m [20]. The China Geodetic Coordinate System 2000 (CGCS2000) is used as a reference frame and BeiDou Time (BDT) is used as a time system. January 6, 1980, 00:00:00, UTC is an initial epoch [21]. Before 2015, BDS used the following frequencies: B1, B2 and B3. After modernization, so after 2015, BDS use: B1-3, B2a, B2b. The $B 3$ frequency is the same as before modernization [19, 22]. The frequencies are presented in Table 1. Figure 1 presents the BDS satellites footprints. The BDS system also consist of three segments, space, ground and user segments. There are 33 BeiDou System 3 satellites in space, and 16 BDS-2 satellites. Some of the BDS-3 satellites are still in the testing phase.

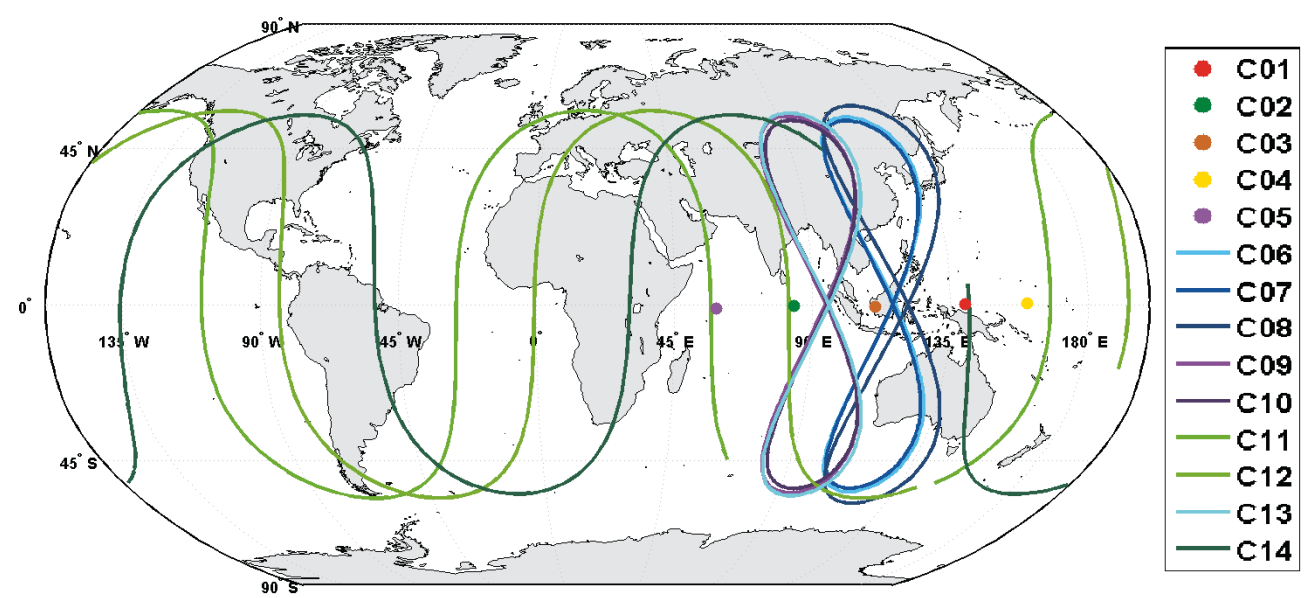

Fig. 1. BeiDou-2 System satellite footprints

The European navigation system is called Galileo, and just like BDS is a civil system unlike GPS or GLONASS. Galileo is expected to be fully operational in 2020. The Galileo satellites are placed on the three circular medium Earth orbits at an altitude of 23,222. The Galileo full constellation will consist of 30 satellites. 24 of those satellites will be operational and 6 satellites will be spare [7]. The inclination of the Galileo system orbits is $56^{\circ}$ [1]. Galileo System Time (GST) is used a time system for Galileo, 22 August 1999 00:00:00 UTC is the start epoch of GST. The Galileo Terrestrial Reference Frame (GTRF) is the reference frame for Galileo [2, 17]. Galileo uses the following signal frequencies: E1, E5a, E5b, E5 and E6 [2]. The frequencies are also shown in Table 1. Figure 2 shows the Galileo footprints. There are 26 Galileo satellites placed on their orbits. Some of them are still in their test phase and will soon be included in the Galileo constellation.

Table 1 shows the frequencies of GPS, Galileo and BDS [19, 22, 23]. 
Table 1. BDS, GPS and Galileo frequencies and signals

\begin{tabular}{||c|c|c|c|c|c|}
\hline \multicolumn{2}{|c|}{ GPS } & \multicolumn{2}{c|}{ Galileo } & \multicolumn{2}{c|}{ BDS } \\
\hline Signal & $\begin{array}{c}\text { Frequency } \\
{[\mathrm{MHz}]}\end{array}$ & Signal & $\begin{array}{c}\text { Frequency } \\
{[\mathrm{MHz}]}\end{array}$ & Signal & $\begin{array}{c}\text { Frequency } \\
{[\mathrm{MHz}]}\end{array}$ \\
\hline \hline L1 & 1575.42 & E1 & 1575.42 & B1-3 & 1575.42 \\
\hline L2 & 1227.60 & - & - & - & - \\
\hline L5 & 1176.45 & E5a & 1176.45 & B2a & 1176.45 \\
\hline- & - & E5b & 1207.14 & B2b & 1207.140 \\
\hline- & - & E5 & 1191.795 & B2-3 & 1191.795 \\
\hline- & - & E6 & 1278.75 & - & - \\
\hline- & - & - & - & B1 & 1561.098 \\
\hline- & - & - & - & B2 & 1207.14 \\
\hline- & - & - & - & B3 & 1268.52 \\
\hline
\end{tabular}

Source: own study based on [22, 23]

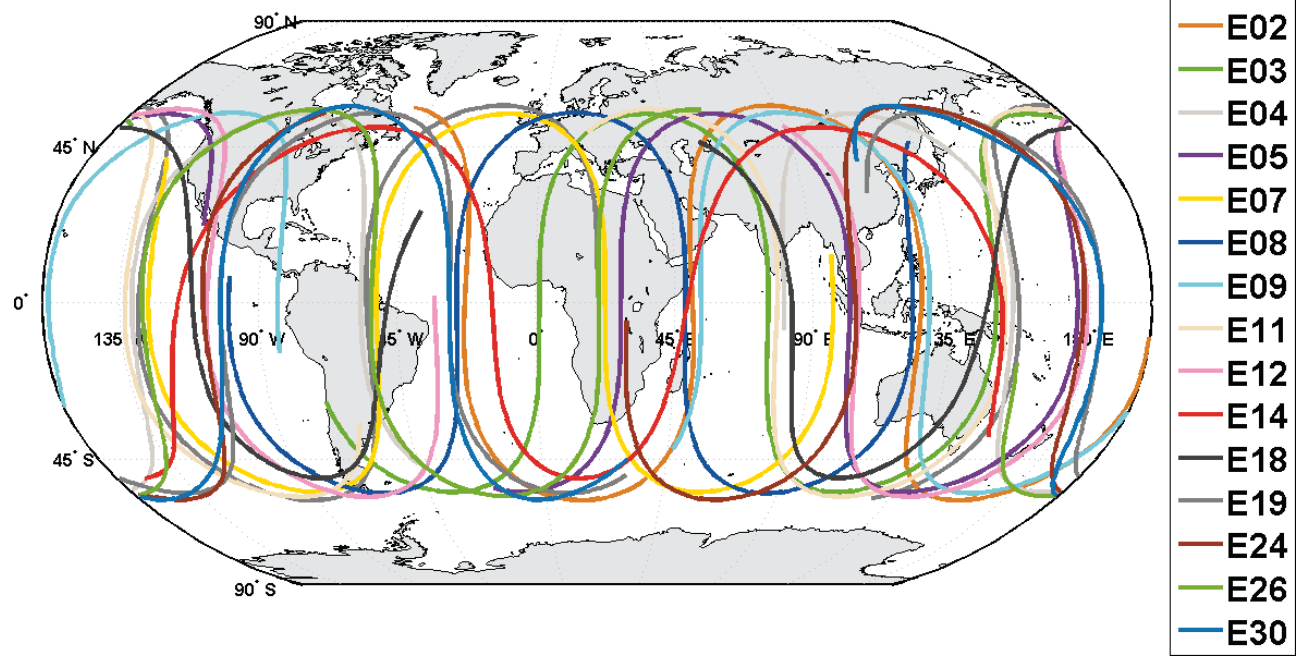

Fig. 2. Galileo System satellite footprints

\section{SPP and Inter-System Bias}

The pseudo-range between a receiver and a satellite determined by the signal travel time is used in the Single Point Positioning (SPP) method. To obtain the pseudo-range, the following Equation (1) can be used [15, 24]:

$$
D=\rho-\left(\mathrm{ISB}+c \delta t_{u}\right)+c \delta t_{s}+d_{T}+d_{I}+\varepsilon_{\rho}
$$


where:

$D$ - pseudorange,

$\rho$ - geometric distance between satellite and receiver,

$c \delta t_{s}$ - satellite clock offset scaled by the speed of light,

$c \delta t_{u}$ - receiver clock offset scaled by the speed of light,

$d_{T}$ - tropospheric delay,

$d_{I}$ - ionospheric delay,

$\varepsilon_{\mathrm{Q}}$ - other unmodelled errors.

The $c \delta t_{s^{\prime}} d_{T^{\prime}} d_{I}$ are computed and incorporated into $D$ term. The $c \delta t_{u}$ is included in ISB (Inter-System bias) term.

The ISBsys (merged with receiver clock offset) and the three-dimensional coordinates of receiver is estimated [15]. To create a design matrix $A$ in the part of experiment where the Inter-System Bias was taken into account, we use Equation (2):

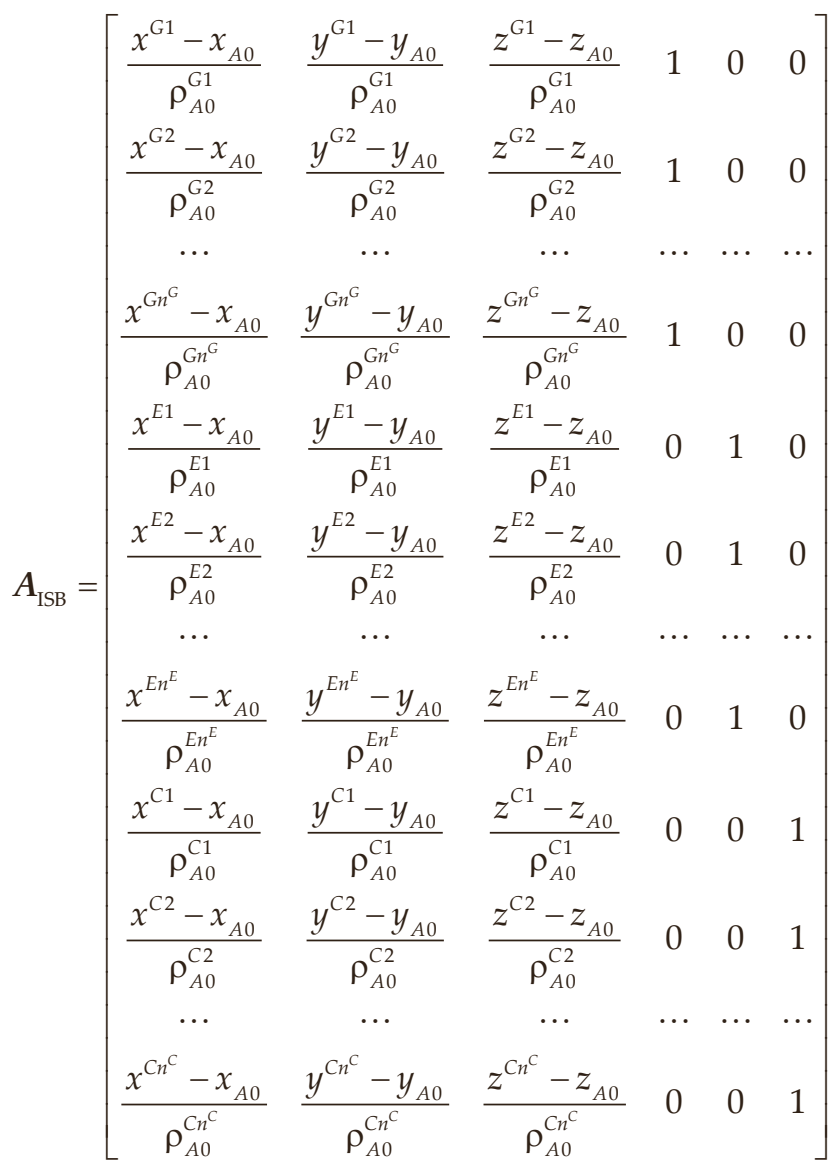


When the ISB was not taken into account, the $A$ matrix was created as follows:

$$
A_{\mathrm{noISB}}\left[\begin{array}{cccc}
\frac{x^{G 1}-x_{A 0}}{\rho_{A 0}^{G 1}} & \frac{y^{G 1}-y_{A 0}}{\rho_{A 0}^{G 1}} & \frac{z^{G 1}-z_{A 0}}{\rho_{A 0}^{G 1}} & 1 \\
\frac{x^{G 2}-x_{A 0}}{\rho_{A 0}^{G 2}} & \frac{y^{G 2}-y_{A 0}}{\rho_{A 0}^{G 2}} & \frac{z^{G 2}-z_{A 0}}{\rho_{A 0}^{G 2}} & 1 \\
\cdots & \cdots & \cdots & \cdots \\
\frac{x^{G n^{G}}-x_{A 0}}{\rho_{A 0}^{G n^{G}}} & \frac{y^{G n^{G}}-y_{A 0}}{\rho_{A 0}^{G n^{G}}} & \frac{z^{G n^{G}}-z_{A 0}}{\rho_{A 0}^{G n^{G}}} & 1 \\
\frac{x^{E 1}-x_{A 0}}{\rho_{A 0}^{E 1}} & \frac{y^{E 1}-y_{A 0}}{\rho_{A 0}^{E 1}} & \frac{z^{E 1}-z_{A 0}}{\rho_{A 0}^{E 1}} & 1 \\
\cdots & \frac{y^{E 2}-y_{A 0}}{\rho_{A 0}^{E 2}} & \frac{z^{E 2}-z_{A 0}}{\rho_{A 0}^{E 2}} & 1 \\
\frac{x^{E n^{E}}-x_{A 0}}{\rho_{A 0}^{E n^{E}}} & \frac{y^{E n^{E}}-y_{A 0}}{\rho_{A 0}^{E n^{E}}} & \frac{z^{E n^{E}}-z_{A 0}}{\rho_{A 0}^{E n^{E}}} & 1 \\
\frac{x^{C 1}-x_{A 0}}{\rho_{A 0}^{C 1}} & \frac{y^{C 1}-y_{A 0}}{\rho_{A 0}^{C 1}} & \frac{z^{C 1}-z_{A 0}}{\rho_{A 0}^{C 1}} & 1 \\
\frac{x^{C 2}-x_{A 0}}{\rho_{A 0}^{C 2}} & \frac{y^{C 2}-y_{A 0}}{\rho_{A 0}^{C 2}} & \frac{z^{C 2}-z_{A 0}}{\rho_{A 0}^{C 2}} & 1 \\
\cdots & \frac{y^{C n^{C}}-y_{A 0}}{\rho_{A 0}^{C n^{C}}} & \frac{z^{C n^{C}}-z_{A 0}}{\rho_{A 0}^{C n^{C}}} & 1 \\
\frac{x_{A 0}^{C}}{\rho^{C 2}} & 1
\end{array}\right]
$$

where in (2) and (3): $x y z^{C}, x y z^{E}, x y z^{G}$ are BDS, Galileo and GPS satellites coordinates, $\rho_{A 0}^{G}, \rho_{A 0}^{E}, \rho_{A 0}^{C}$ are geometric distances between approximate point position and satellites, $n$ stands for the number of observable satellites, $x y z_{A 0}$ are approximate point coordinates. Columns four, five and six in the Equation (2) are used to estimate receiver clock error and Inter-System bias. In the following approach, the receiver clock is absorbed by code biases during the parameter estimation process [11]. During the de-correlation process, the bias for code observations may occur. The ISB should be taken into the account during the positioning with different GNSS systems. In the case of Equation (3), the ISB is not estimated. The presented design matrix $A$ is used in absolute SPP positioning where the least squares estimation method is used

$$
\boldsymbol{X}=\left(\boldsymbol{A}^{T} \boldsymbol{P} A\right)^{-1}\left(\boldsymbol{A}^{T} \boldsymbol{P L}\right)
$$


In Equation (4) $\boldsymbol{P}$ is a weight matrix presented in further part of paper, $L$ is a free terms matrix and $A$ is a design matrix. By solving this equation (4) we get increments to coordinates $\Delta x, \Delta y$ and $\Delta z$ and a clock error $\Delta t$. Matrix $X$ looks like follows:

$$
\boldsymbol{X}=\left[\begin{array}{c}
\Delta x \\
\Delta y \\
\Delta z \\
\Delta t
\end{array}\right]
$$

\section{Experiment Description}

In the experiment, data from four GNSS stations were used with three different receivers (two stations WTZ2 and WROC use the same receivers). The JFNG station was equipped with the TRIMBLE NETR9 5.22 receiver, the WROC station use the LEICA GR50 4.11/7.102 receiver. The same receiver is used by the WTZ2 station. The NNOR station uses the SEPT POLARX4 2.9.5-extref1 receiver. The data was obtained at 5 minute intervals. GPS P1 observations were used, for Galileo E1 observations were used and for BDS B1 observations were used. The ionospheric and tropospheric corrections were modelled. For tropospheric corrections, the Vienna Mapping Function (VMF1) was used [25]. The Technical University of Catalonia ionosphere maps were used for ionosphere correction. The precise orbits in sp3 format were obtained and used during the tests. In the experiment the two different approaches were compared. In the first one, the Intersystem Bias was not taken into the account, and the one receiver clock offset was estimated for all three GNSS systems: GPS, BDS and Galileo. In the second approach, the parameter which includes receiver clock offset and ISB was estimated for each system separately. The values of this parameter were compared. The results of the SPP between these two approaches were also compared to see whether the estimation of ISB has an influence on the received position. To determine the accuracy of positioning, the obtained SPP positions were compared to GNSS stations coordinates. The obtained biases were transformed into a local topocentric coordinate system and presented. The weight matrix $\boldsymbol{P}$ was created as follows:

$$
\boldsymbol{P}=\left[\begin{array}{cccc}
\frac{\sin \left(E l_{1}\right)^{2}}{\sigma^{2}} & 0 & 0 & 0 \\
0 & \frac{\sin \left(E l_{2}\right)^{2}}{\sigma^{2}} & 0 & 0 \\
0 & 0 & \cdots & 0 \\
0 & 0 & 0 & \frac{\sin \left(E l_{n}\right)^{2}}{\sigma^{2}}
\end{array}\right]
$$


where $E l_{i}$ is a satellite elevation of each satellite in given epoch, $\sigma$ stands for a code observation standard deviation (STD). For BDS and GPS, the STD was set to $0.6 \mathrm{~m}$ and $0.3 \mathrm{~m}$ for Galileo [10]. Elevation mask was set to the $5^{\circ}$.

All tests were performed using self-made software in MatLab. Software reads RINEX 2 and RINEX 3, sp3 files with precise clocks and satellites orbits, as well as other necessary data. All the computations were performed using self-made software.

\section{Experiment Results}

The Figure 3 shows the number of satellites available during the experiment for each station. It is visible that the number of available BDS satellites is higher in the NNOR and JFNG stations than in the case of GPS when on stations WROC and WTZ2 number of BDS satellites is comparable with number of Galileo satellites, a result of the BDS constellation characteristics. The common number of satellites for WROC and WTZ2 stations is about 15 when for stations JFNG and WROC number of common satellites hovers around 20. Figure 4 shows the TDOP factor values observed on each station. It can be observed that on each station, the TDOP factor for Galileo is the highest. It was probably caused by the low number of available satellites for this system. For conjoined clocks, the value of TDOP is the lowest.

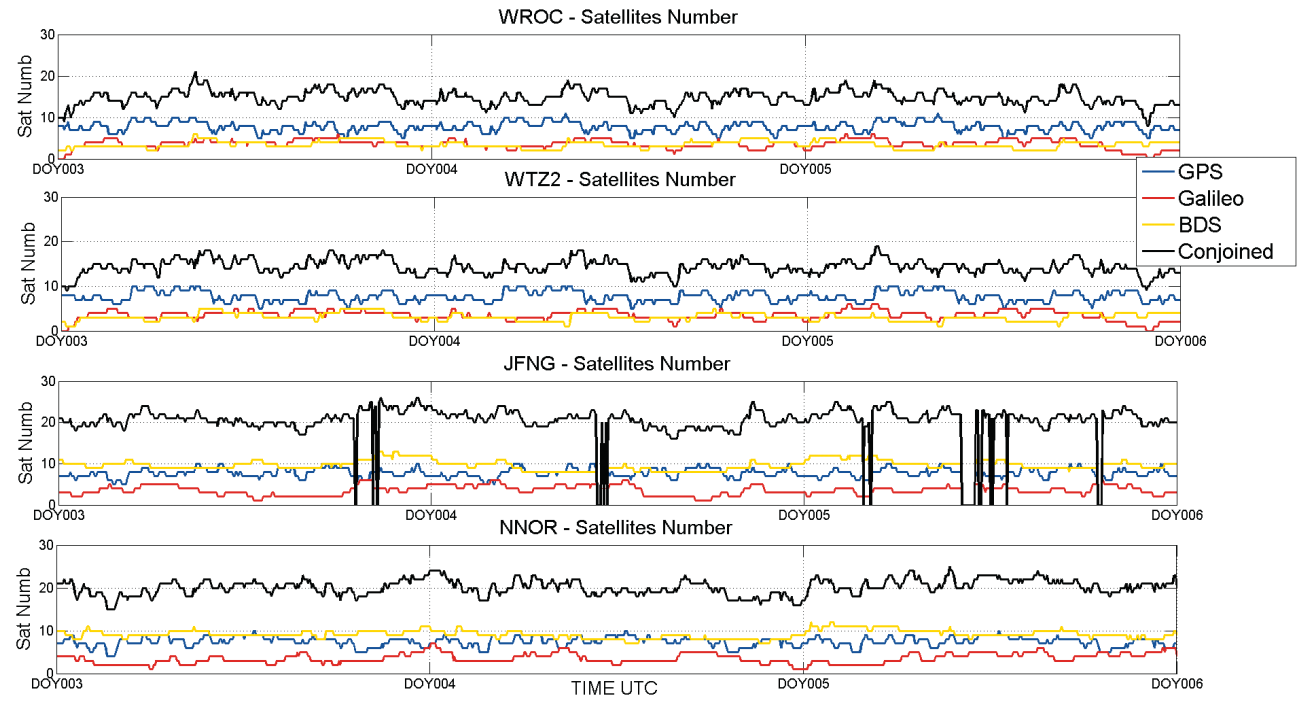

Fig. 3. Number of satellites during experiment 

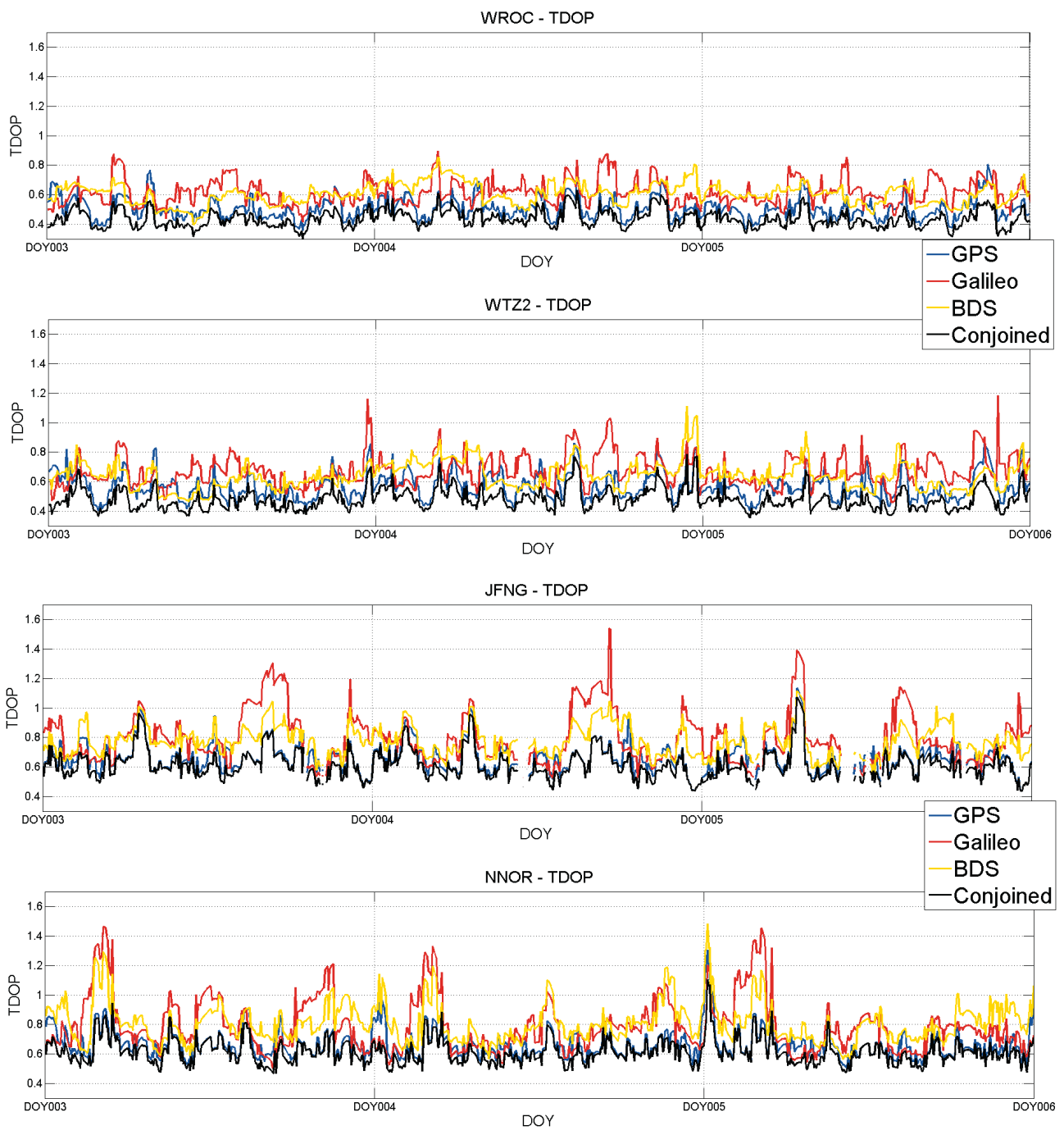

Fig. 4. TDOP factor

Figure 5 shows the results of the receivers clock offset estimation. For each receiver a correlation between offsets for GPS, Galileo and BDS is noticeable. The differences between each estimated offset are the Inter-System bias and the differences between systems are not constant because of noises. The receiver clock offset estimated jointly has noticeable correlation with the results obtained for each system separately. However, it behaves less stably that the offset estimated as parameter including ISB. For the LEICA GR50 4.11/7.102 receivers the values of estimated receiver clock offsets are different, however it is noticeable that they act similarly. There is no visible correlation between number of satellites and the estimated clock error. 
However, we can observe a correlation between TDOP factor values and estimated clock offsets. When the TDOP factor values increase, we can observe significant changes in the estimated offset values, regardless of the GNSS system.
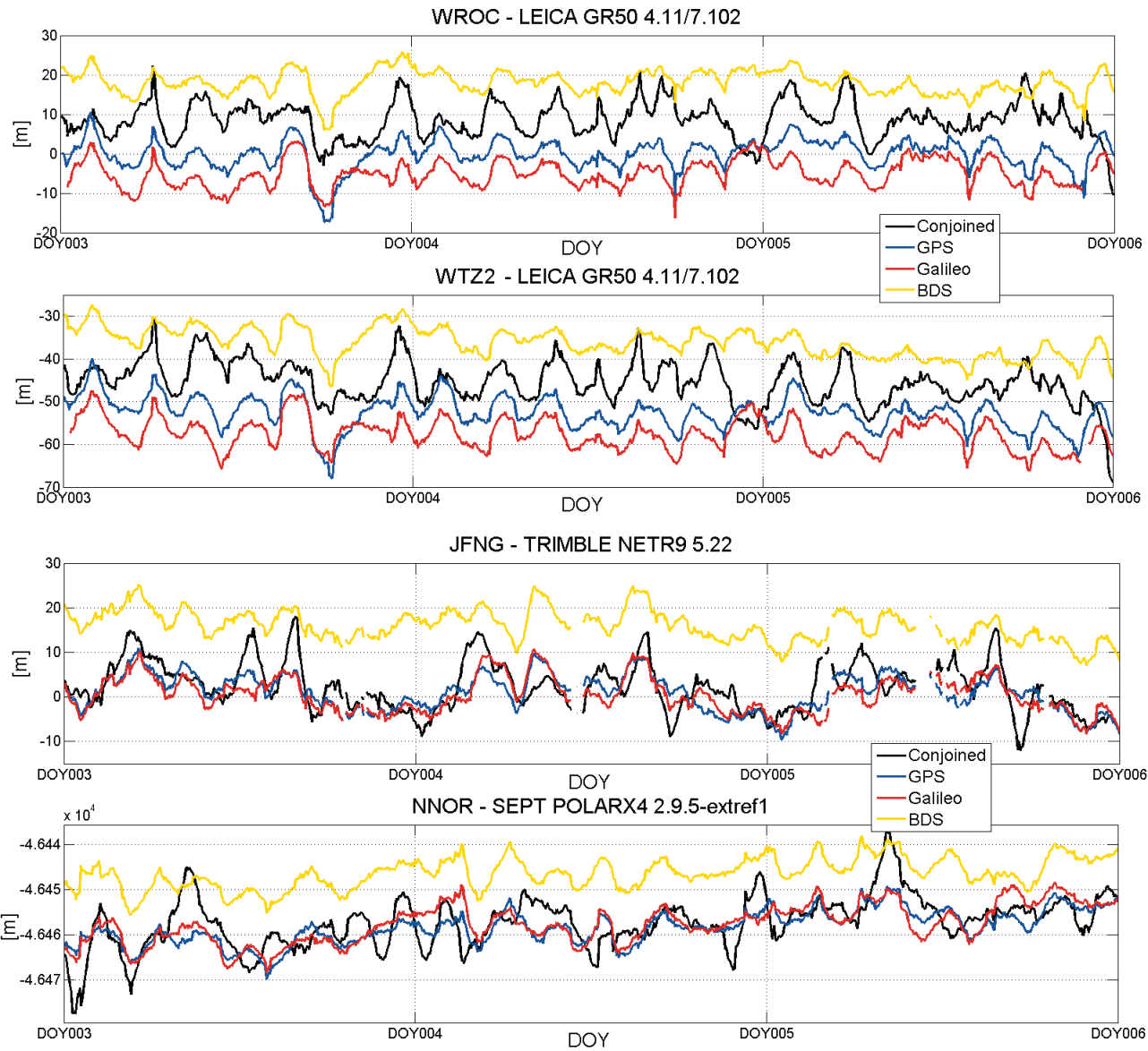

Fig. 5. Estimated receivers clock offsets

Table 2 shows the results of the SPP positioning when estimating ISB and without it. It is clearly visible that results obtained with estimated ISB are more precise and accurate. This is especially visible for the up direction in the local topocentric coordinate system, where the obtained mean and standard deviation (STD) values are much lower that for the approach where the ISB is not taken into account. The vertical mean results seem to be similarly accurate, but the STD values show that results when ISB is taken into account are more accurate. 
Table 2. Single Point Positioning results

\begin{tabular}{|c|c|c|c|c|c|c|c|c|}
\hline & \multicolumn{8}{|c|}{ ISB taken into account } \\
\hline & \multicolumn{2}{|c|}{ JFNG } & \multicolumn{2}{|c|}{ WROC } & \multicolumn{2}{|c|}{ WTZ2 } & \multicolumn{2}{|c|}{ NNOR } \\
\hline & Mean [m] & Std [m] & Mean [m] & Std $[\mathrm{m}]$ & Mean $[\mathrm{m}]$ & Std [m] & Mean $[\mathrm{m}]$ & Std $[\mathrm{m}]$ \\
\hline $\mathrm{dN}$ & -0.36 & 1.83 & 1.25 & 2.10 & 1.20 & 2.29 & 0.42 & 1.69 \\
\hline $\mathrm{dE}$ & 1.70 & 1.05 & 1.54 & 1.26 & 0.35 & 1.23 & 2.20 & 1.14 \\
\hline \multirow[t]{4}{*}{$\mathrm{dU}$} & -0.11 & 2.97 & -100 & 4.91 & -1.79 & 4.94 & 0.04 & 3.68 \\
\hline & \multicolumn{8}{|c|}{ ISB not taken into account } \\
\hline & \multicolumn{2}{|c|}{ JFNG } & \multicolumn{2}{|c|}{ WROC } & \multicolumn{2}{|c|}{ WTZ2 } & \multicolumn{2}{|c|}{ NNOR } \\
\hline & Mean [m] & Std [m] & Mean [m] & Std $[\mathrm{m}]$ & Mean [m] & Std [m] & Mean [m] & Std [m] \\
\hline $\mathrm{dN}$ & 6.43 & 2.83 & -0.72 & 3.55 & -0.40 & 3.35 & -4.82 & 2.70 \\
\hline $\mathrm{dE}$ & 2.48 & 2.25 & -1.72 & 2.29 & -2.02 & 2.30 & 2.68 & 1.91 \\
\hline $\mathrm{dU}$ & -7.42 & 6.19 & 9.14 & 7.70 & 7.12 & 7.43 & -6.00 & 6.51 \\
\hline
\end{tabular}

Source: own study based on MatLab results

\section{Summary and Conclusions}

In this study, the need to take into account the Inter-System Bias was presented and evaluated. The experiment used three day long data from 4 GNSS stations: WTZ2, WROC (equipped with the same type of the receiver), JFNG and NNOR. The results from each station are presented.

By taking into account Inter-System Bias, both positioning accuracy and availability are improved. For Single Point Positioning, the obtained results are more accurate and precise when the receiver clock offsets including ISB were estimated for each GPS, Galileo and BDS separately that when there was only one receiver clock offset estimated for all three systems. In the experiment it was also shown that there is a correlation between estimated clocks for GPS, Galileo and BDS separately and the difference in the values is an Inter-System Bias. Estimating ISB allows for more precise positioning results to be obtained for car-navigation or GIS purposes.

\section{Acknowledgment}

This research was supported by grant with agreement no. UMO-2017/27/N/ ST10/00412 from National Science Center, Poland.

\section{References}

[1] Cai C., Luo X., Liu Z., Xiao Q.: Galileo Signal and Positioning Performance Analysis Based on Four IOV Satellites. The Journal of Navigation, vol. 67(5), 2014, pp. 810-824. https://doi.org/10.1017/S037346331400023X.

[2] Paziewski J., Wielgosz P.: Assessment of GPS+Galileo and multi-frequency Galileo single-epoch precise positioning with network corrections. GPS Solutions, vol. 18, 2013, pp. 571-579. https://doi.org/10.1007/s10291-013-0355-3. 
[3] Odolinski R., Teunissen P.J.G., Odijk D.: First combined COMPASS/BeiDou-2 and GPS positioning results in Australia. Part I: singlereceiver and relative code-only positioning. Journal of Spatial Science, vol. 59, issue 1, 2014, pp. 3-24. https://doi.org/10.1080/14498596.2013.840865.

[4] Cai C., Gao Y., Pan L., Zhu J.: Precise point positioning with quad-constellations: GPS, BeiDou, GLONASS and Galileo. Advances in Space Research, vol. 56, issue 1, 2015, pp. 133-143. https://doi.org/10.1016/j.asr.2015.04.001.

[5] Cai C., He C., Santerre R., Pan L., Cui X., Zhu J.: A comparative analysis of measurement noise and multipath for four constellations: GPS, BeiDou, GLONASS and Galileo. Survey Review, vol. 48, issue 349, 2016, pp. 287-295. https://doi. org/10.1179/1752270615Y.0000000032.

[6] Ochieng W., Sauer K., Cross P., Sheridan K., Iliffe J., Lannelongue S., Ammour N., Petit K.: Potential Performance Levels of a Combined Galileo GPS Navigation System. The Journal of Navigation, vol. 54, issue 2, 2001, pp. 185-197. https://doi.org/10.1017/S037346330100131X.

[7] Li X., Ge X., Dai X., Ren M., Fritsche M., Wickert J., Schuh H.: Accuracy and reliability of multi-GNSS real-time precise positioning: GPS, GLONASS, BeiDou, and Galileo. Journal of Geodesy, vol. 89, 2015, pp. 607-635. https://doi.org/10.1007/s00190-015-0802-8.

[8] Li X., Zhang X., Ren X., Fritsche M., Wickert J., Schuh H.: Precise positioning with current multi-constellation Global Navigation Satellite Systems: GPS, GLONASS, Galileo and BeiDou. Scientific Reports, vol. 5, 2015, 8328. https://doi.org/10.1038/srep08328.

[9] Tegedor J., Øvstedal O., Vigen E.: Precise orbit determination and point positioning using GPS, Glonass, Galileo and BeiDou. Journal of Geodetic Science, vol. 4, 2014, pp. 65-73.

[10] Pan L., Cai C., Santerre R., Zhang X.: Performance evaluation of single-frequency point positioning with GPS, GLONASS, BeiDou and Galileo. Survey Review, vol. 49, issue 354, 2016, pp. 197-205. https://doi.org/10.1080/00396265.2016. 1151628.

[11] Pan L., Zhang X. Li X., Li X., Lu C., Liu J., Wang Q.: Satellite availability and point positioning accuracy evaluation on a global scale for integration of GPS, GLONASS, BeiDou and Galileo. Advances in Space Research, vol. 63, issue 9, 2019, pp. 2696-2710. https://doi.org/10.1016/j.asr.2017.07.029.

[12] Baldysz Z., Nykiel G., Figurski M.: Analysis of the impact of Galileo observations on the tropospheric delays estimation. [in:] 2017 Baltic Geodetic Congress (BGC Geomatics), Gdansk, Poland, 22-25 June 2017, pp. 65-71. https://doi.org/10.13140/ RG.2.2.19456.64001.

[13] Nykiel G., Figurski M.: Impact of Galileo Observations on the Position and Ambiguities Estimation of GNSS Reference Stations. [in:] 2017 Baltic Geodetic Congress (BGC Geomatics), Gdansk, Poland, 22-25 June 2017, pp. 225-231. https://doi. org/10.1109/BGC.Geomatics.2017.11. 
[14] Cai C., Gao Y., Pan L., Dai W.: An analysis on combined GPS/COMPASS data quality and its effect on single point positioning accuracy under different observing conditions. Advances in Space Research, vol. 54, issue 5, 2014, pp. 818-829. https://doi.org/10.1016/j.asr.2013.02.019.

[15] Angrisano A., Gaglione S., Gioia C., Massaro M., Troisi S.: Benefit of the NeQuick Galileo Version in GNSS Single-Point Positioning. International Journal of Navigation and Observation, 2013, 302947. https://doi.org/10.1155/2013/302947.

[16] Leick A., Rapoport L., Tatarnikov D.: GPS Satellite Surveying. $4^{\text {th }}$ ed. John Wiley \& Sons, Hoboken 2015.

[17] Chu F-Y., Yang M.: GPS/Galileo long baseline computation: method and performance analyses. GPS Solutions, vol. 18, 2013, pp. 263-272. https://doi. org/10.1007/s10291-013-0327-7.

[18] Lee Y.C.: Analysis of range and position comparison methods as ameans to provide GPS integrity in the user receiver. [in:] Institute of Navigation, Annual Meeting, 42nd, Seattle, WA, June 24-26, 1986, Proceedings, Institute of Navigation, Washington 1986, pp. 1-4.

[19] Zhang X., Wu M., Liu W., Li X., Yu S., Lu C., Wickert J.: Initial assessment of the COMPASS/BeiDou-3: new-generation navigation signals. Journal of Geodesy, vol. 91, 2017, pp. 1225-1240. https://doi.org/10.1007/s00190-017-1020-3.

[20] He L., Ge M., Wang J., Wickert J.; Schuh H..: Experimental Study on the Precise Orbit Determination of the BeiDou Navigation Satellite System. Sensors, vol. 13(3), 2013, pp. 2911-2928. https://doi.org/10.3390/s130302911.

[21] Deng C., Tang W., Liu J., Shi C.: Reliable single-epoch ambiguity resolution for short baselines using combined GPS/BeiDou system. GPS Solutions, vol. 18, 2014, pp. 375-386. https://doi.org/10.1007/s10291-013-0337-5.

[22] Montenbruck O., Hauschild A., Steigenberger P., Hugentobler U., Teunissen P., Nakamura S.: Initial assessment of the COMPASS/BeiDou-2 regional navigation satellite system. GPS Solutions, vol. 17, 2013, pp. 211-222. https://doi. org/10.1007/s10291-012-0272-x.

[23] Kwaśniak D., Cellmer S., Nowel K.: Precise positioning in Europe using the Galileo and GPS combination. [in:] The 10th International Conference: Environmental engineering: 10 ${ }^{\text {th }}$ ICEE: Selected papers, Vilnius Gediminas Technical University Press, Vilnius 2017. https://doi.org/10.3846/enviro.2017.210.

[24] Parkinson B., Axelrad P.: Autonomous GPS integrity monitoring using the pseudorange residual. Navigation: Journal of the Institute of Navigation, vol. 35(2), 1988, pp. 255-274.

[25] Urquhart L., Santos M., Nievinski F., Bohm J.: Generation and Assessment of VMF1 Type Grids Using North American Numerical Weather Models. [in:] Rizos Ch., Willis P. (eds.), Earth on the Edge: Science for a Sustainable Planet: Proceedings of the IAG General Assembly, Melbourne, Australia, June 28 - July 2, 2011, International Association of Geodesy Symposia, vol. 139, Springer Berlin Heidelberg, 2016, pp. 3-9. 